\title{
Research on Application of Wind/PV Hybrid System in Outdoor lighting
}

\author{
Bing Li \\ School of Mechanical Engineering, Baicheng Normal University, Baicheng City, Jilin province, 137000, \\ China
}

Keywords: Wind/PV Hybrid; Outdoor lighting; Application; Expressway

\begin{abstract}
With the energy crisis approaching, new energy has become one of the major energy sources in the world, and the Wind/PV Hybrid System have came into being. Wind power generation and solar cell array power generation are commonly referred to as Wind/PV Hybrid Lighting Systems, and their role is to solve the existing problems in lighting systems. Therefore, this has been the favor of many people in the industry. Based on the author's learning and practical experience, the present situation of Wind/PV Hybrid Lighting Systems was firstly studied, and then the composition of Wind/PV Hybrid Lighting Systems was analyzed. Finally, the application of Wind/PV Hybrid Generation Systems in expressway was discussed.
\end{abstract}

\section{Introduction}

The hybrid of wind energy and solar energy in time and space makes up for the defects of wind power and photovoltaic power generation system. This confirms that wind power generation is more efficient than single wind power or solar power generation. In recent years, with the construction of highway in China, highway service level and highway mileage in China are also constantly improved. At the same time, higher requirements are also put forward for equipment and power supply system along the expressway. Therefore, the power supply system along the expressway faces the new direction of power supply. Wind power generation system combines the advantages of solar energy and wind energy, and makes a comprehensive use of these two, so as to further promote the highway power supply system toward a new energy system.

\section{Wind/PV Hybrid Lighting Systems Status}

Ever since Danish scholars proposed the idea of Wind/PV Hybrid Generation in 1981, many researches have been carried out in this field both at home and abroad, and many important achievements have been made. In recent years, the research has focused on the optimal design of the system and the rational allocation of resources. Some foreign research institutions launched some large-scale software to calculate the optimal configuration of the cloud hybrid system through simulation system performance parameters, power supply costs and power matching method and energy matching method. The most powerful and outstanding one is the Hybrid2 software, which was cooperated and developed by Us Renewable Energy Research Laboratory. In the wind and light hybrid optimization matching calculation and system control, many research institutes in China have also carried out a great deal of research work, and made some technical breakthroughs. At present, China's off grid Wind/PV Hybrid Generation Systems technology is relatively mature, and this has been widely used in the power grid coverage of remote villages, communication base stations, street lamps, border posts, remote areas of independent power supply system. The system matching is optimized by weather data, solar daily radiation, wind frequency distribution, geographical condition and load condition. The representative system matching include: installed $100 \mathrm{~K} \mathrm{~W}$ Tibet, Shuanghu wind and light hybrid power station; and wind and light hybrid power supply system for whole process monitoring of the Guangzhou Southern China express line, Hunan Shao Yong (Shaoyang to Yongzhou) and other expressways; wind and light hybrid street lamps in 
Shanghai, Tianjin, Changsha, Erdos and other places; and Bohai oil platform wind and light hybrid power supply system, Honghu wind and light hybrid fishing boat power supply system.

\section{Composition of Wind/PV Hybrid Lighting Systems}

The overall structure of the wind and light hybrid power generation system is shown in figure 1 . The system is composed of 3 parts: the generation of electric energy, the control of electric energy conversion and the storage and consumption of energy.

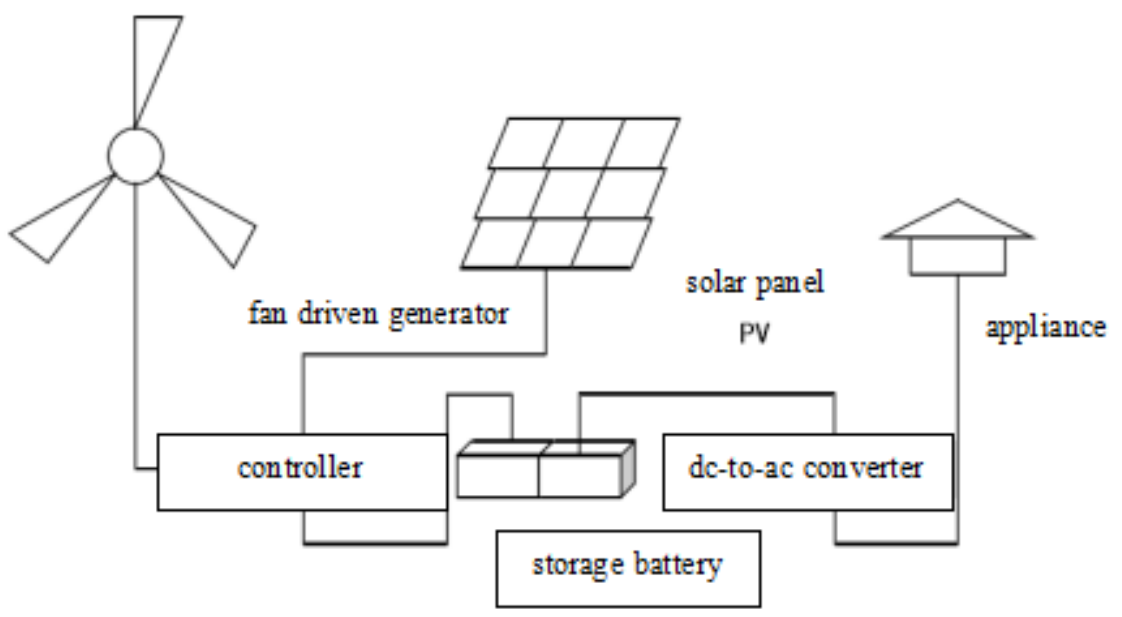

Fig. 1 Structure of Wind/PV Hybrid Generation Systems

Electrical energy generation link. Electrical energy generation involves two parts: wind power and solar power generation. Part of the wind power generation can be converted into electrical energy through a DC fan or an AC fan; solar power generation uses solar panels to convert light energy into electrical energy.

Power conversion control link. The control section of power conversion is composed of DC or DC converter, main control circuit and so on. It is the core part of the power generation system. The three-phase alternating current output by the AC fan needs to be rectified into the DC or DC converter. The direct current fan outputs direct current and is sent directly to the DC or DC converter after voltage regulation; the DC output from the solar panel is usually passed through 1 anti diode and then fed into the DC or DC converter. The main control circuit usually adopts PLC or MCU, DSP and other control chips, and the power conversion is realized by controlling the DC or DC converter. At the same time, it can also collect and process all kinds of information and parameters so as to realize the functions of equipment protection, risk warning and so on.

Energy storage and consumption link. The energy storage section includes storage and consumption in two parts. The storage of electric energy is partly carried by the battery, which is used to eliminate the imbalance of energy supply and demand caused by weather and plays a role in regulating and balancing the load in the whole system. The power consumption is mainly composed of DC load and AC load. The DC load can be directly introduced by the battery, or the required DC voltage can also be supplied by 1 step-up or step-down DC conversion circuits; as for AC loads, the DC output from the battery needs to be converted into alternating current.

\section{Application of Wind/PV Hybrid Generation Systems on Expressway}

\section{Power supply status of expressway electromechanical system}

At present, the power supply of the highway electromechanical system is still using the traditional power supply mode. Namely, in the system, the power distribution center is equipped with power supply and distribution facilities, and the power is transmitted by the power cable. The transformer converts the medium voltage from the substation into low voltage electricity. After the power distribution cabinet is centralized and distributed, the power supply is supplied to the power 
equipment along the expressway through the power cable. The premise of the traditional power supply mode of the electromechanical system of expressway is that the electric equipment should be centralized as much as possible, otherwise the load center can not be formed, and the power supply and distribution facilities can not be configured. The toll system and communication system on the highway are generally built at toll stations or toll management centers, which can form a centralized power load. In this case, the traditional power supply mode is more suitable. As for the outfield electromechanical equipment and road lighting facilities on the expressway, they are scattered along both sides of the road, and the distance between the equipment is very far, which makes the load center of the power supply system not prominent. They need to be laid long power cables to achieve the purpose of power supply for the equipment, so the power consumption is serious, resulting in high cost of power supply and can not guarantee the quality of power supply.

Disadvantages of Traditional power supply mode adopted by highway lighting equipment. First, it increases construction costs. With the traditional power supply mode, a large number of power cables should be laid along the expressway. Long pipe laying, huge amount of the engineering, great wasting of manpower and material bring great construction costs. In addition, the current highway is gradually developing into the mountain area, which requires the power supply system to build a remote dedicated medium voltage power supply circuit for power supply, so that the cost of power supply construction of the expressway increases year by year. Second, it affects normal operation. The traditional power supply mode mainly uses the copper core cable to supply power. At present, the price of copper in the market is relatively high, and the power cables along the highway are relatively easy to steal. Therefore, cable theft is very easy to occur, resulting in loss of property on the expressway. After the power cable is stolen, it is difficult to confirm and re lay the cable stolen points, which makes the electrical equipment near the point of theft not working properly in a certain period of time. This will also lead that the operation of the highway units can not grasp the vehicle running along the situation. This not only has a great impact on the highway safety, rapid and efficient operation, but also increases the maintenance cost of power supply system and the instability of power supply. Thirdly, there exists security problem. Highway mileage is longer, so the highway electromechanical equipment is generally distributed along the road. This requires a long line. Along with the extension of the pipeline, the voltage loss of the grid will be serious, so it is necessary to build the power distribution and variable voltage system along the expressway to avoid the voltage loss of the power grid. This not only increases the distribution cost of the project, but also impossibly ask some people on duty at any time to maintain due to it belongs to the field power supply, so there are great security risks. Fourth, it waste energy. The outfield monitoring equipment of Expressway usually runs 24 hours without interruption. Although the power of each individual device is not large, the power consumption is very large due to the large number of monitoring equipment along the expressway. Therefore, the traditional mode of power supply is not conducive to the economic operation of the highway.

Application prospect of Wind/PV Hybrid Generation Systems in expressway electromechanical system. Wind/PV Hybrid Generation Systems has simple structure, strong mobility and less power. The utility model can provide stable and continuous current output for low power electrical equipment, and is especially suitable for field monitoring equipment and road lighting facilities. The wind and light hybrid power generation system is free from maintenance after the installation is completed, and it can save the operating cost of traditional power supply mode. Moreover, the quality of power supply will be greatly improved compared with the traditional power supply mode.

\section{Summary}

Wind/PV Hybrid Generation Systems make the effective and rational use of wind and solar energy and other new clean energy. This not only saves a lot of conventional energy, making the operation cost greatly reduced, but also bring better economic and social benefits. Practice showed that Wind/PV Hybrid Generation Systems can utilize the natural hybrid characteristics of wind energy and solar energy to provide more stable power supply for the power equipment along the 
expressway. The system can also allocate the capacity of the system reasonably and effectively according to the actual power consumption and resource conditions of the equipment, which can reduce the cost of the power generation system while ensuring the reliability of the system power supply. With the wind and light hybrid generation technology continues to mature, the Wind/PV Hybrid Generation Systems will be more widely applied in the field of highway.

\section{References}

[1] Feng Wei, Zhang Jingzhu, Yang Aiqiong, Zuo Hong, Li Rui. Application of Wind and Light Complementary Power Supply System in Automatic Weather Station[J]. Communication World, 2015, (11): 51-52.

[2] Yin Guiliang, Li Xiangnan, Guo Lei, Li Xiaoliang. Application of Hybrid Energy Storage System in Wind and Light Complementary Micro Grid[J]. Journal of Power System and Automation, 2015, (01): 49-53+59.

[3] Xu Zheng, Guang Rui, Wu Zhenlong. Investigation and Analysis of Application Efficiency of Wind and Light Complementary Street Lamps[J]. Solar Energy, 2014, (05): 21-25.

[4] Hu Chenlong. Research on the Application of Wind and Light Complementary Street Lamps in Large Scale Science and Technology Parks[J]. Digital Technology and Application, 2013, (02): 82.

[5] Meng, Chen Yang, Lv Yuxin, Hao Ying, Dong Lei. Application of Wind Power and Light Complementary Independent Power Supply System in Communication Base Station[J]. Microcomputer and Applications, 2012, (01): 67-69+72.

[6] Zhang Junling, Yin Jianying, Dang Zheng. Wind and Light Complementary Generation System and Application[J]. Energy Research and Utilization, 2011, (04): 48-49.

[7] Wei Lumin, Xiong Weijian. Application of Wind and Light Complementary Power Generation Technology in Street Lighting[J]. Energy Technology, 2009, (03): 162-163. 